\title{
Identification of potential transcriptomic markers in developing pediatric sepsis: a weighted gene co-expression network analysis and a case-control validation study
}

\author{
Yiping $\mathrm{Li}^{1 \dagger}$, Yanhong $\mathrm{Li}^{1,2 \dagger}$, Zhenjiang Bai ${ }^{3 \dagger}$, Jian Pan ${ }^{1}$, Jian Wang ${ }^{1 *}$ and Fang Fang ${ }^{1 *}$ (1)
}

\begin{abstract}
Background: Sepsis represents a complex disease with the dysregulated inflammatory response and high mortality rate. The goal of this study was to identify potential transcriptomic markers in developing pediatric sepsis by a coexpression module analysis of the transcriptomic dataset.

Methods: Using the R software and Bioconductor packages, we performed a weighted gene co-expression network analysis to identify co-expression modules significantly associated with pediatric sepsis. Functional interpretation (gene ontology and pathway analysis) and enrichment analysis with known transcription factors and microRNAs of the identified candidate modules were then performed. In modules significantly associated with sepsis, the intramodular analysis was further performed and "hub genes" were identified and validated by quantitative real-time PCR (qPCR) in this study.

Results: 15 co-expression modules in total were detected, and four modules ("midnight blue", "cyan", "brown", and "tan") were most significantly associated with pediatric sepsis and suggested as potential sepsis-associated modules. Gene ontology analysis and pathway analysis revealed that these four modules strongly associated with immune response. Three of the four sepsis-associated modules were also enriched with known transcription factors (false discovery rate-adjusted $P<0.05$ ). Hub genes were identified in each of the four modules. Four of the identified hub genes (MYB proto-oncogene like 1, killer cell lectin like receptor G1, stomatin, and membrane spanning 4-domains A4A) were further validated to be differentially expressed between septic children and controls by qPCR.
\end{abstract}

Conclusions: Four pediatric sepsis-associated co-expression modules were identified in this study. qPCR results suggest that hub genes in these modules are potential transcriptomic markers for pediatric sepsis diagnosis. These results provide novel insights into the pathogenesis of pediatric sepsis and promote the generation of diagnostic gene sets.

Keywords: Transcriptomic markers, Co-expression modules, Pediatric sepsis, Diagnosis, Hub genes

\section{Background}

Sepsis represents a complex disease with the dysregulated inflammatory response and high mortality rate. It is the world's leading killer of children [1]. However, current

\footnotetext{
*Correspondence: wj196312@vip.163.com; baseff77@hotmail.com ${ }^{\dagger}$ Yiping Li, Yanhong Li and Zhenjiang Bai contributed equally to this work ${ }^{1}$ Institute of Pediatric Research, Children's Hospital of Soochow University, Suzhou, China

Full list of author information is available at the end of the article
}

knowledge of the pathogenesis of sepsis is limited $[2,3]$.

In the past decade, several studies have reported the transcriptional profiling of sepsis using microarrays to identify candidate genes involved in sepsis development [4-7]. Co-expression module analysis of transcriptomic dataset has the likelihood of discovering robust candidates for diagnosis and treatment. Therefore, we investigated gene expression patterns between pediatric sepsis patients and healthy controls in this study based on public microarray dataset. Network construction and module 
detection were performed. The importance of candidate modules identified in this study were evaluated, and modules most significantly associated with sepsis were further interpreted by enrichment analysis, intramodular analysis and quantitative real-time PCR (qPCR).

To carry out these analyses, we used the $\mathrm{R}$ software (v3.3.2) [8] and Bioconductor packages [9] for data pre-procession and weighted gene co-expression network analysis. Functional interpretation and network construction of co-expression modules were also performed using DAVID [10, 11] and Cytoscape [12] software, respectively. Enrichment analysis of the candidate module genes with known transcription factors and microRNAs was also performed using WebGestalt [13]. Validation of gene expression patterns was performed by qPCR in this study.

\section{Methods}

\section{Microarray datasets search and selection}

In this study, we searched public microarray datasets till Jul 12, 2016, according to the keywords "sepsis" in Gene Expression Omnibus (GEO) database [14]. The datasets obtained were further selected for subsequent analysis, and our selection criteria were: (a) case-control dataset; (b) dataset using whole blood from children for gene expression analysis; (c) dataset providing detailed gene expression data; (d) dataset with sample size (septic children and controls in total) larger than 100. Animal studies and studies of adults were excluded.

\section{Pre-procession of microarray gene expression dataset}

One dataset fulfilled the selection criteria and was used for further analysis. This eligible dataset (GSE13904) was generated using the Affymetrix Human Genome U133 Plus 2.0 Array from 99 pediatric sepsis patients (32 sepsis and 67 septic shock patients) and 18 normal controls [7]. Raw data saved in.CEL files of the eligible dataset was downloaded from GEO database, and then pre-processed (background correction, quantile normalization, $\log 2$ transformed) using the Robust Multichip Average (RMA) method of the R package "affy" [15]. Next, the hybridization probes were mapped to genes (Entrez IDs) according to the platform table. Probes mapping to multiple genes and probes not mapping to genes were excluded. When multiple probes mapped to the same gene, arithmetic

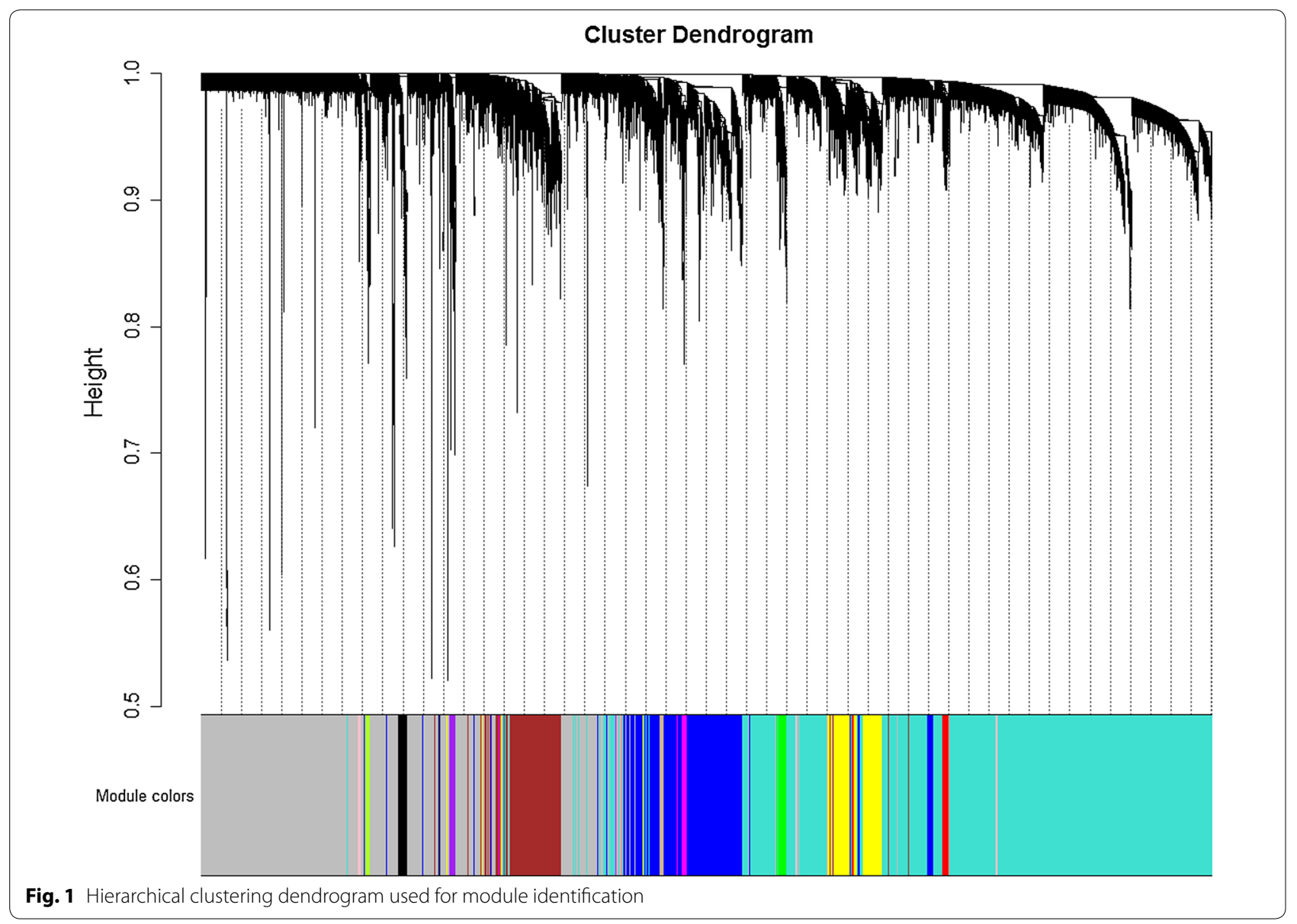


Table 1 Top 4 sepsis-associated co-expression modules

\begin{tabular}{lccr}
\hline Module & Correlation coefficient with pediatric sepsis & $\boldsymbol{P}^{\mathbf{a}}$ & Number of genes \\
\hline Midnight blue & -0.67 & $2 \times 10^{-16}$ & 39 \\
Cyan & 0.65 & $2 \times 10^{-15}$ & 39 \\
Brown & 0.54 & $4 \times 10^{-10}$ & 1382 \\
Tan & -0.53 & $1 \times 10^{-9}$ & 80
\end{tabular}

a Student asymptotic $P$ value for correlation
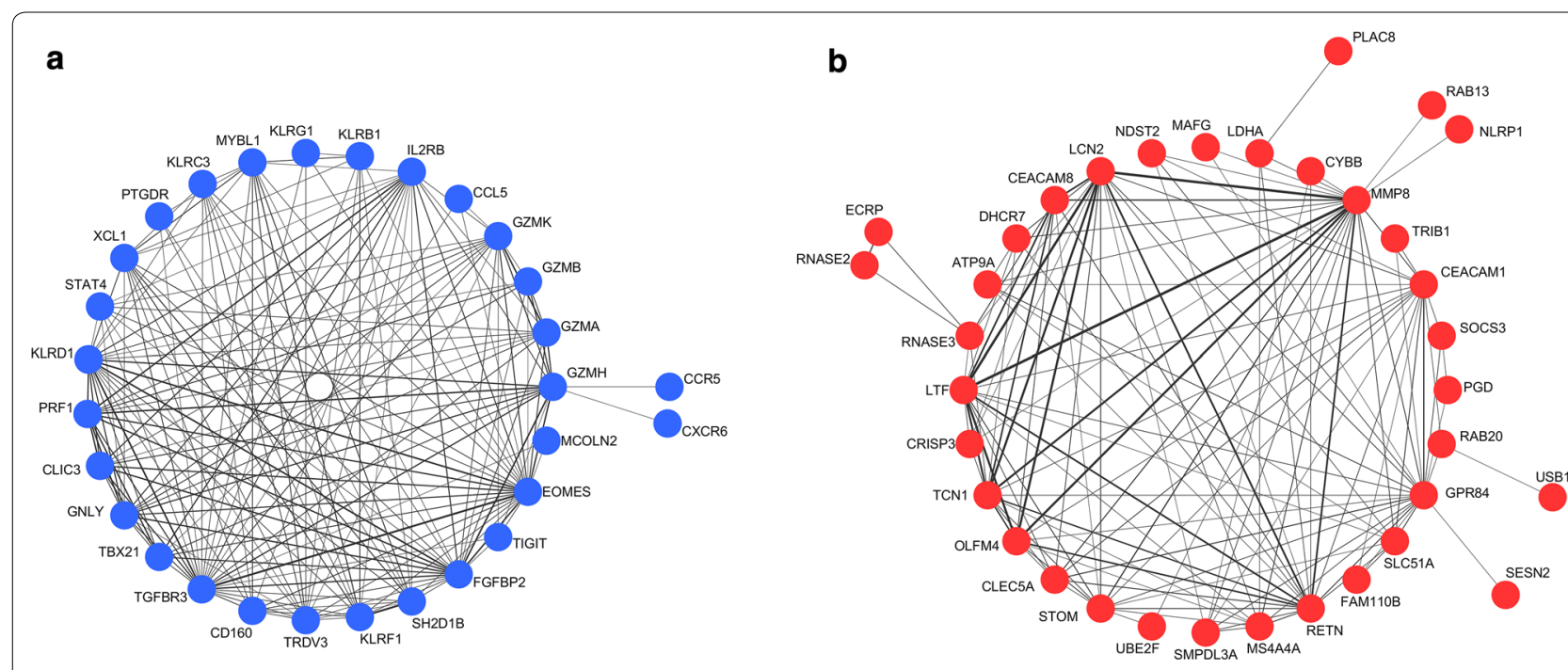

Fig. 2 Co-expression networks of the top 2 sepsis-associated modules. a Network of module "midnight blue". b Network of module "cyan". Edge widths are proportional to the correlation coefficients

mean of probe values were calculated to represent gene expression.

\section{Weighted gene co-expression network analysis}

Weighted gene co-expression network analysis was carried out using the R package "WGCNA" [16] in this study. Pre-processed gene expression data were first checked for missing values and outliers. The genes and samples which passed the test were collected for network analysis. One-step network construction and module detection were then performed, with the soft-thresholding power $\beta$ set to 14 according to the criterion of approximate scalefree topology (Additional file 1), the minimum module size set to 30, and the threshold for merging of modules set to 0.25 . Modules significantly associated with sepsis were identified based on the correlation between module eigengenes and sample types (sepsis patients versus healthy controls). Gene relationships to sepsis and modules were then evaluated by gene significance (GS, correlation of individual gene expression with sepsis) and module membership (MM, correlation of individual gene expression with module eigengene). Network construction of co-expression modules was also performed using Cytoscape 3.4.0 [12] software.

\section{Enrichment analysis}

Functional interpretation [gene ontology (GO) analysis and Kyoto Encyclopedia of Genes and Genomes (KEGG) pathway analysis] of the co-expression genes in sepsisassociated modules was further performed using DAVID $6.8[10,11]$. In GO analysis, a $P$ value threshold of 0.05 was used to identify significantly enriched GO terms [17]. In pathway analysis, enrichment analysis was carried out using the hypergeometric test with a $P$ value threshold of 0.05 based on the KEGG database [18]. Enrichment analysis of the candidate module genes with known transcription factors and microRNAs was also performed using WebGestalt [13], according to the criteria: (a) false discovery rate (FDR)-adjusted $P$ value $<0.05$; (b) a minimum number of genes in a category: two. 


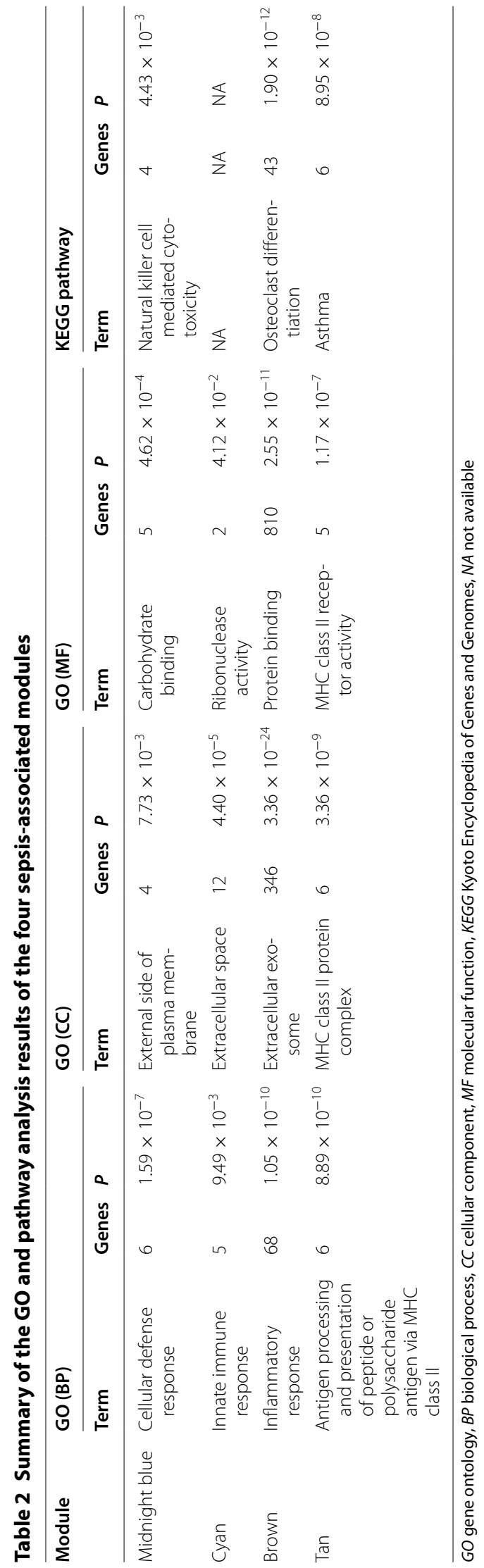


Table 3 Summary of the transcription factor enrichment analysis results of the four sepsis-associated modules

\begin{tabular}{llll}
\hline Module $^{\text {a }}$ & Transcription factor & Official full name & FDR-adjusted $\boldsymbol{P}$ value \\
\hline Midnight blue & ETS2 & ETS proto-oncogene 2, transcription factor & $1.64 \times 10^{-2}$ \\
Brown $^{\text {b }}$ & ETS2 & ETS proto-oncogene 2, transcription factor & $5.85 \times 10^{-8}$ \\
& ELF1 & E74 like ETS transcription factor 1 & $9.52 \times 10^{-6}$ \\
& SPI1 & Spi-1 proto-oncogene & $4.37 \times 10^{-5}$ \\
& ETV4 & ETS variant 4 & $3.86 \times 10^{-4}$ \\
RUNX1 & Runt related transcription factor 1 & $1.37 \times 10^{-3}$ \\
SRF & Serum response factor & $1.37 \times 10^{-3}$ \\
& GABPA & GA binding protein transcription factor alpha subunit & $2.51 \times 10^{-3}$ \\
& JUN & Jun proto-oncogene, AP-1 transcription factor subunit & $3.07 \times 10^{-3}$ \\
ETV7 & ETS variant 7 & $3.07 \times 10^{-3}$ \\
Tan & Interferon regulatory transcription factor family & $8.03 \times 10^{-3}$ \\
& SREBF1 & Sterol regulatory element binding transcription factor 1 & $9.52 \times 10^{-3}$ \\
& IRF1 & Interferon regulatory factor 1 & $4.03 \times 10^{-2}$ \\
& POU5F1 & POU class 5 homeobox 1 & $4.03 \times 10^{-2}$ \\
\hline
\end{tabular}

FDR false discovery rate

a No significantly enriched transcription factor was identified in module "cyan"

b Transcription factors with FDR-adjusted $P$ value $<0.01$ were listed for module "brown" for the sake of brevity

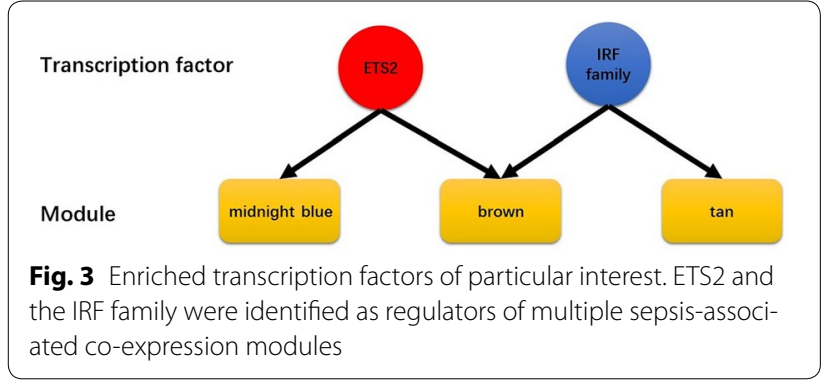

Intramodular analysis and quantitative real-time PCR

For modules significantly associated with sepsis, the intramodular analysis was performed and "hub genes" were identified according to the criteria: (a) LOG10 ( $P$ value of GS) $\geq 10$; (b) $M M \geq 0.8$.

Validation of hub gene expression patterns was performed by qPCR in this study. 45 pediatric sepsis patients were included. 16 children who were scheduled for minor elective surgery such as circumcision or inguinal hernia repair were also included as the control group. All heparinized blood samples were obtained from Children's Hospital of Soochow University. Informed consent was obtained from each participating individual's guardian. The study procedure was approved by the ethics committee of Children's Hospital of Soochow University.

Mononuclear cells (MNCs) were isolated, and then stored at $-80^{\circ} \mathrm{C}$ before RNA extraction. Total RNA was extracted using RNAiso (TaKaRa, Dalian, China). The RNA was reverse-transcribed using oligo- $\mathrm{dT}$, and mouse mammary tumor virus reverse transcriptase. qPCR was performed with SYBR Green master mix. Primers designed were shown in Additional file 2. Gene expression was normalized to $\beta$-actin mRNA. The relative expression of gene transcript was calculated using the $2^{-\Delta \Delta C t}$ method. Comparison of clinical characteristics between study groups was performed using the MannWhitney U test for continuous variables and the Fisher's exact test for categorical variables. Mann-Whitney U test was also performed to determine the expression difference between septic children and the control group. Statistical analyses were performed with GraphPad Prism software (GraphPad Software Inc.). All $P$ values are twosided. $P<0.05$ was considered as statistically significant.

In addition, the diagnostic performance of hub genes were also evaluated in the validation group ( 45 septic children and 16 controls) by receiver operating characteristic (ROC) curve plotting and area under the curve (AUC) values calculation using the R package "pROC" [19].

\section{Results}

Co-expression modules in pediatric sepsis development

Original search identified one eligible microarray dataset (GSE13904) [7]. Pre-processing of this dataset resulted in expression data of 20,464 genes in 99 pediatric sepsis samples and 18 normal controls. According to the parameters (soft-thresholding power $\beta=14$, minimum 
module size $=30$, and the threshold for merging of modules $=0.25)$ and the hierarchical clustering dendrogram (Fig. 1) used for the module identification in this study, 15 modules in total were detected with sizes ranging from 8359 to 39 genes. Among the 15 candidate modules, four modules (module "midnight blue", module "cyan", module "brown", and module "tan") were most significantly associated with sepsis and suggested as potential sepsis-associated modules (see Table 1). Co-expression networks of the top 2 sepsis-associated modules (module "midnight blue" and module "cyan") are shown in Fig. 2.

\section{Enrichment analysis results}

Advanced analyses (GO analysis and pathway analysis) were carried out for further functional investigation of the four sepsis-associated co-expression modules. Table 2 presented a summary of the GO and pathway analysis results. In the $\mathrm{GO}$ analysis, the top $\mathrm{GO}$ biological process terms enriched are "cellular defense response" in module "midnight blue", "innate immune response" in module "cyan", "inflammatory response" in module "brown", and "antigen processing and presentation of peptide or polysaccharide antigen via MHC class II" in module "tan". In the pathway analysis, the most significant pathways identified were natural killer cell-mediated cytotoxicity in module "midnight blue", osteoclast differentiation in module "brown", and asthma in module "tan", when we mapped the module genes to the KEGG database. No significantly enriched pathway was identified in module "cyan".

Enrichment analysis of genes in the four sepsis-associated modules with known transcription factors and microRNAs was also performed using WebGestalt [13]. As presented in Table 3, three of the four sepsisassociated modules were enriched with known transcription factors (FDR-adjusted $P$ value $<0.05$ ). Among them, transcription factors of particular interest are ETS proto-oncogene 2, transcription factor (ETS2) and the
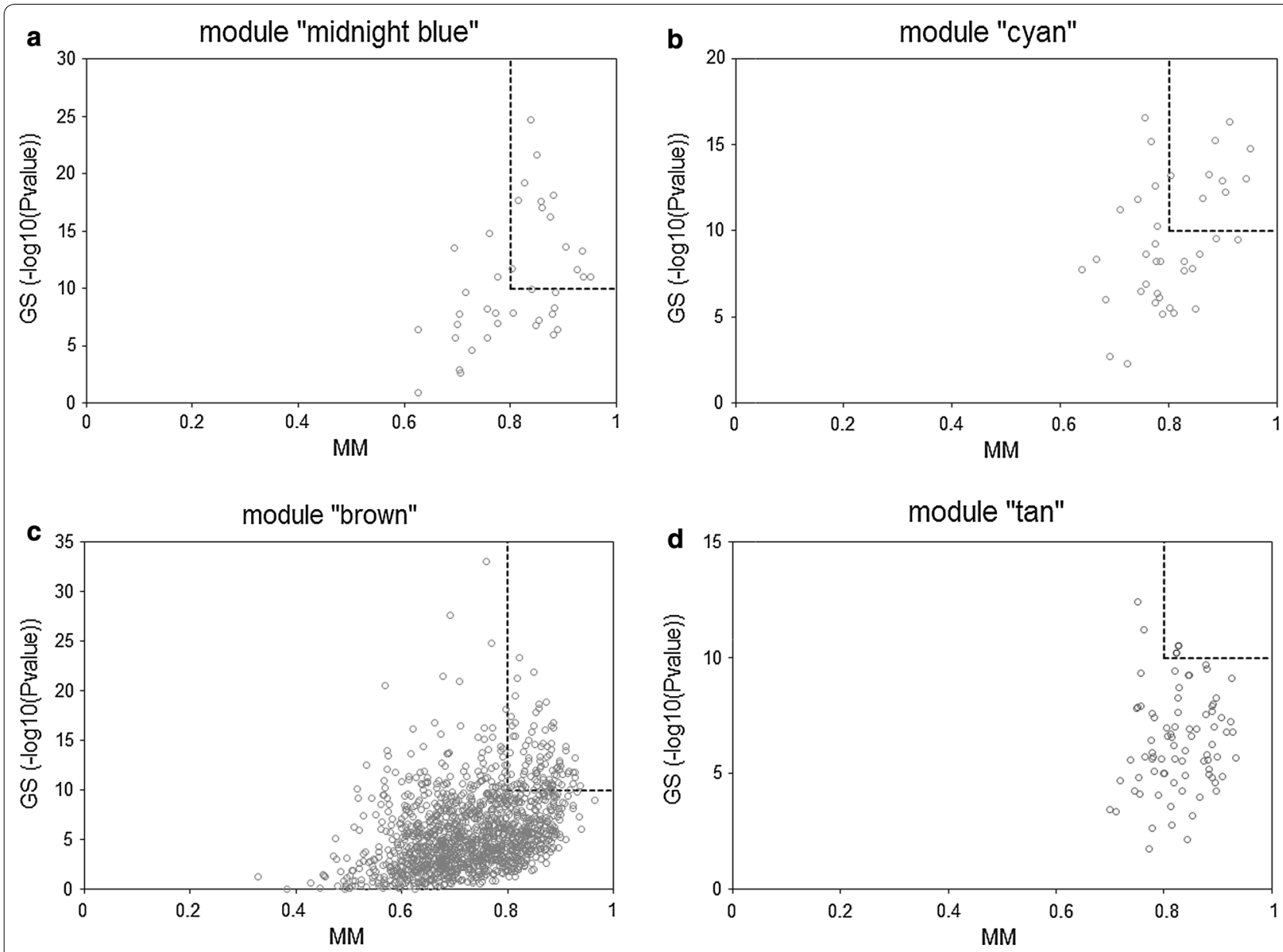

Fig. 4 Scatterplots of the intramodular analysis results and hub genes identified in the four sepsis-associated modules. a Scatterplot for module "midnight blue". b Scatterplot for module "cyan". c Scatterplot for module "brown". d Scatterplot for module "tan". GS gene significance, MM module membership 
interferon regulatory transcription factor (IRF) family, as they function in the regulation of multiple sepsisassociated co-expression modules (see Fig. 3). However, no significantly enriched microRNA was identified in this study.

\section{Hub gene identification and validation}

The intramodular analysis was performed in the four sepsis-associated co-expression modules, and hub genes were identified in each of the four modules. As shown in Fig. 4, 14, 9, 98, and two hub genes were identified within module "midnight blue", "cyan", "brown", and "tan" respectively. Significant changes in expression of those hub genes were detected between 99 pediatric sepsis samples and 18 normal controls from dataset GSE13904 [7] (Fig. 5). four of the identified hub genes [MYB protooncogene like 1 (MYBL1) and killer cell lectin-like receptor G1 (KLRG1) form module "midnight blue", stomatin $(S T O M)$ and membrane spanning 4-domains A4A (MS4A4A) from module "cyan"], predicted to be potential biomarkers for pediatric sepsis in intramodular analysis and with little known in sepsis, were further assessed for their difference in expression between 45 septic children and 16 controls (Table 4) by qPCR. As presented in Fig. 6, the expression levels of MYBL1 and KLRG1 in the sepsis group were significantly lower than those of the control group $(P<0.001$ respectively, see Fig. 6a, b). While $S T O M$ and $M S 4 A 4 A$ were significantly overexpressed in sepsis samples, compared with controls $(P=0.04$ and $P<0.001$ respectively, see Fig. $6 \mathrm{c}, \mathrm{d})$. As for diagnostic prediction quality, the four hub genes showed good performance as well according to the ROC analysis in the validation group (45 septic children and 16 controls) (see Fig. 7). Both the qPCR results and ROC analysis results suggest that the four hub genes (MYBL1, KLRG1, STOM and $M S 4 A 4 A$ ) could be novel diagnostic biomarkers for pediatric sepsis.

\section{Discussion}

Some genes have been reported to be up-regulated or down-regulated in pediatric sepsis patients $[5,7]$. Identification of the most important candidate genes and pathways involved in sepsis pathogenesis is a challenge currently. Growing high-throughput transcriptomic data
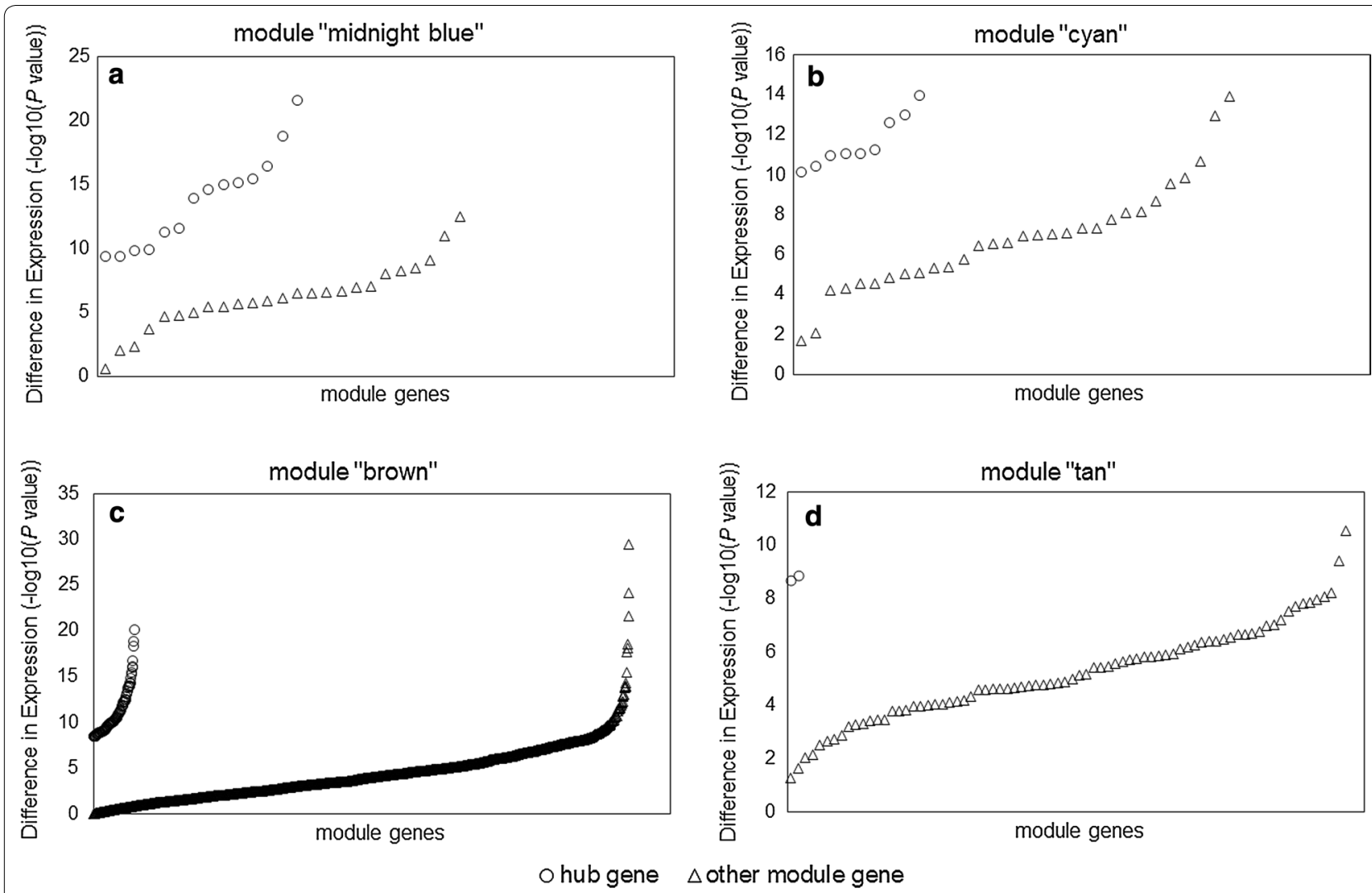

Fig. 5 Differences in expression of hub genes and other module genes between 99 pediatric sepsis samples and 18 normal controls from dataset GSE13904. a Differences in expression for module "midnight blue". b Differences in expression for module "cyan". c Differences in expression for module "brown". d Differences in expression for module "tan" 
Table 4 Clinical characteristics of the validation group

\begin{tabular}{llll}
\hline Characteristic & Sepsis & Control & $P$ \\
\hline Number & 45 & 16 & \\
Age, median years [range] & $1.50[0.08-13.42]$ & $1.92[0.08-10.50]$ & $0.95^{\mathrm{a}}$ \\
Gender & & & $0.22^{\mathrm{b}}$ \\
$\quad$ Male & 27 & 13 & \\
Female & 18 & 3 & - \\
Infection site & & & - \\
Lung (\%) & $15(33.3)$ & - & - \\
Brain (\%) & $11(24.4)$ & - & - \\
Others (\%) & $19(42.2)$ & - & - \\
Septic shock (\%) & $17(37.8)$ & - & - \\
ICU stay, median days & $5.38[0.08,30.00]$ & - & \\
[range] & & - & \\
ICU mortality (\%) & $13(28.9)$ & - &
\end{tabular}

a $P$ value of the Mann-Whitney $\mathrm{U}$ test

b $P$ value of the Fisher's exact test

enables weighted gene co-expression network analysis of microarray datasets which has the likelihood of discovering robust candidates for diagnosis and treatment. Hence in this study, we performed a weighted gene coexpression network analysis of public microarray dataset to identify potential transcriptomic markers in developing pediatric sepsis.

In this analysis, 15 co-expression modules were identified, among which four modules (module "midnight blue", module "cyan", module "brown", module "tan") were significantly associated with pediatric sepsis and were potential sepsis-associated modules. The enrichment analysis indicated that transcription factors (ETS2 and the IRF family) play roles in the regulation of multiple sepsis-associated modules. So far there is increasing evidence that the IRF family plays a part in sepsis [20, 21]. Whereas little is known about the impact of ETS2 on sepsis and further investigation is needed.

Through intramodular analysis, 123 hub genes in total were identified in the four sepsis-associated coexpression modules, including hub genes known to play roles in sepsis, and hub genes without previous studies in sepsis. Among those novel hub genes, we assessed the expression patterns of MYBL1, KLRG1, $S T O M$ and MS4A4A from the top 2 sepsis-associated modules (module "midnight blue" and module "cyan") by qPCR. Significantly different expressions between pediatric sepsis patients and controls were detected for all four genes, validating the intramodular analysis results. MYBL1 belongs to the MYB family and is

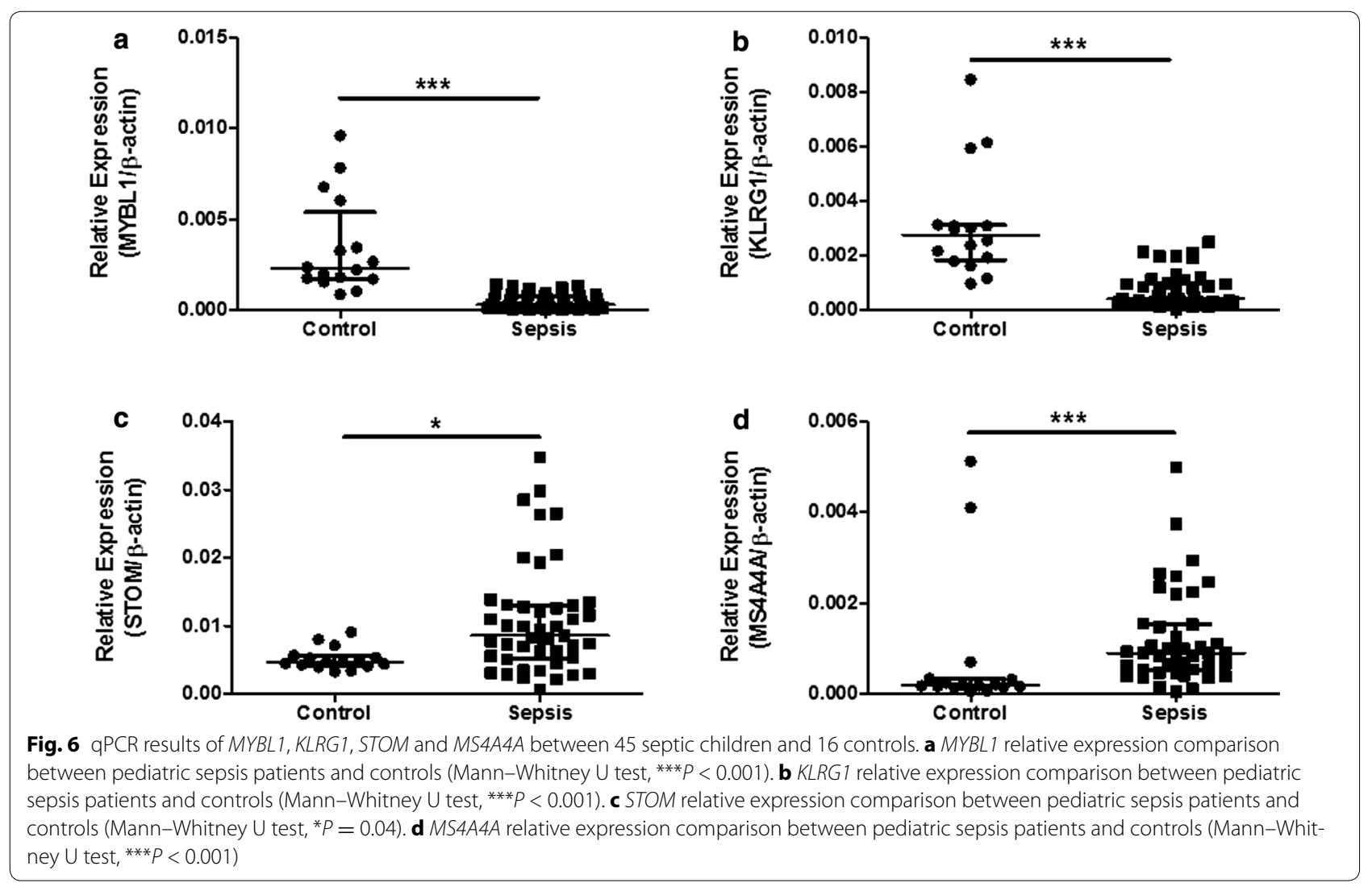



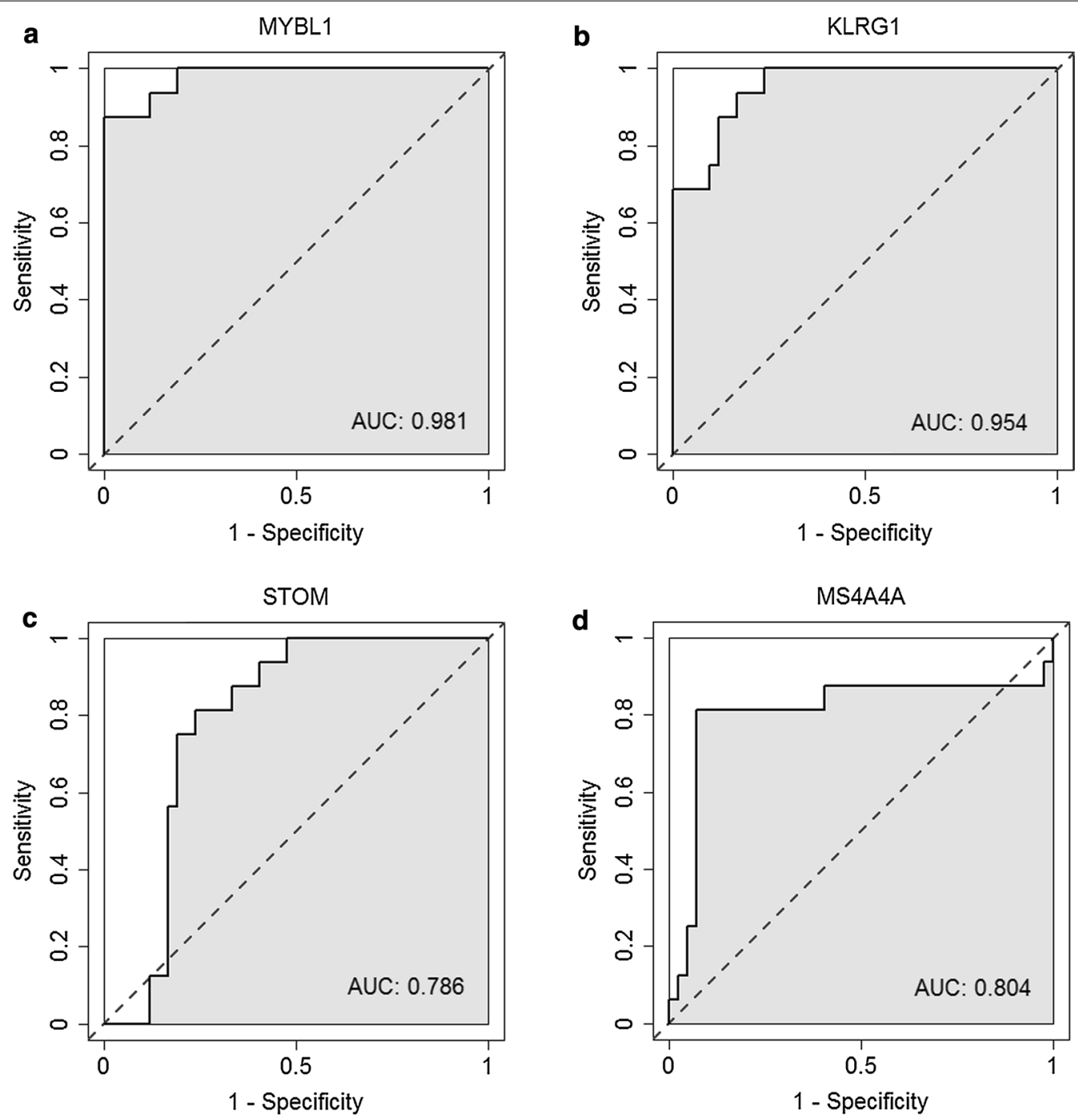

Fig. 7 Receiver operating characteristic (ROC) curves of the hub genes on diagnosis of pediatric sepsis. AUC: area under the ROC curve. a ROC curve of MYBL 1. b ROC curve of KLRG1. c ROC curve of STOM. d ROC curve of MSAA4A

involved in adenoid cystic carcinoma and pediatric glioma [22, 23]. KLRG1 encodes a receptor on antigenexperienced $\mathrm{T}$ cells and natural killer cells $[24,25]$. It is suggested as a senescent marker of human T cells [26]. In tumor microenvironment, KLRG1 is significantly overexpressed in T cells [27]. STOM encodes a major lipid-raft protein stomatin, which locates at the plasma membrane of multiple cell types [28-30], and is associated with non-small cell lung cancer [31] and erbb2 receptor tyrosine kinase 2-positive breast cancer [32]. MS4A4A, a member of the membrane-spanning 4-domains subfamily $A$, is reported to be a cell-surface marker of plasma cells and M2 macrophages [33]. It is also up-regulated in the autopsied brain tissue of Alzheimer's disease patients [34]. In this study, ROC analysis results further indicate that the four hub genes (MYBL1, KLRG1, STOM and MS4A4A) had good diagnostic performance in sepsis, close to that of genes previously reported [35]. Although the exact contributions of the four and other novel hub genes to sepsis are not clear yet, further research is necessary as those genes could be potential transcriptomic markers for sepsis.

Our analysis also has some limitations. The first limitation is the insufficient sample size. A second limitation is the lack of subgroup analyses based on potential influential factors, including age, sex, disease severity and platform usage, considering the reported impact of gender and age on pediatric sepsis patients [36]. The third limitation is the incomplete biological knowledge base 
and pathway information available at present. Hence, to achieve a more convincible conclusion, further analysis using larger sample size is required. Stratified analyses on different factors such as age, gender, disease severity, and platform usage are also needed. Functional studies should be performed as well to address the exact roles of the candidate hub genes in pediatric sepsis.

\section{Conclusions}

In conclusion, we identified four candidate co-expression modules that were differentially expressed between pediatric sepsis patients and normal controls. GO, and pathway analyses revealed that those candidate modules strongly associated with immune response. Transcription factors associated with the modules were also identified through enrichment analysis in this study. qPCR results suggest hub genes (MYBL1, KLRG1, STOM and $M S 4 A 4 A)$ in the candidate modules as promising potential transcriptomic markers for pediatric sepsis diagnosis. To the best of our knowledge, there is no reported weighted gene co-expression network analysis for sepsis so far. We hope this study can help in the diagnosis and treatment of pediatric sepsis.

\section{Additional files}

Additional file 1. Analysis of network topology for candidate soft-thresholding powers ( $\beta \mathrm{s}$ )

Additional file 2. Primers designed for validation of hub gene expression patterns by qPCR.

\begin{abstract}
Abbreviations
AUC: area under the curve; BP: biological process; CC: cellular component; ETS2: ETS proto-oncogene 2, transcription factor; FDR: false discovery rate; GEO: Gene Expression Omnibus; GO: gene ontology; GS: gene significance; ICU: Intensive Care Unit; IRF: interferon regulatory transcription factor; KEGG: Kyoto Encyclopedia of Genes and Genomes; KLRG1: killer cell lectin-like receptor G1; MF: molecular function; MM: module membership; MNCs: mononuclear cells; MS4A4A: membrane spanning 4-domains A4A; MYBL1: MYB proto-oncogene like 1; NA: not available; qPCR: quantitative real-time PCR; RMA: Robust Multichip Average; ROC: receiver operating characteristic; STOM: stomatin.
\end{abstract}

\section{Authors' contributions}

FF and WJ designed the study, analyzed the data and drafted the manuscript; LYP, LYH, and BZJ acquired patient data and performed laboratory experiments; PJ contributed to the study design and writing of the manuscript. All authors read and approved the final manuscript.

\section{Author details}

1 Institute of Pediatric Research, Children's Hospital of Soochow University, Suzhou, China. ${ }^{2}$ Department of Nephrology, Children's Hospital of Soochow University, Suzhou, China. ${ }^{3}$ Pediatric Intensive Care Unit, Children's Hospital of Soochow University, Suzhou, China.

\section{Acknowledgements}

Not applicable.
Competing interests

The authors declare that they have no competing interests.

Availability of data and materials

The datasets supporting the conclusions of this article are within the article and its additional files.

\section{Consent for publication}

All authors approve the publication of this manuscript.

Ethics approval and consent to participate

The study procedure was approved by the ethics committee of Children's Hospital of Soochow University, and informed consent was obtained from each participating individual's guardian.

\section{Funding}

This work was supported by grants from National Natural Science Foundation [Grant Number 81501840]; Jiangsu Provincial Medical Youth Talent [Grant Number QNRC2016768]; Suzhou science and technology development project [Grant Number SYSD2014102]; National Natural Science Foundation [Grant Numbers 81501700, 81571551, 81570125]; Jiangsu province's science and technology support program (Social Development) [Grant Number BE2016675]; and Major International (Regional) Joint Research Project [Grant Number 81420108022$]$. None of the sponsors was involved in the design of the study, in the collection, analysis, and interpretation of data, or in writing the manuscript.

\section{Publisher's Note}

Springer Nature remains neutral with regard to jurisdictional claims in published maps and institutional affiliations.

Received: 17 October 2017 Accepted: 8 December 2017

Published online: 13 December 2017

\section{References}

1. Kissoon N, Carapetis J. Pediatric sepsis in the developing world. J Infect. 2015;71(Suppl 1):S21-6.

2. Stearns-Kurosawa DJ, Osuchowski MF, Valentine C, Kurosawa S, Remick DG. The pathogenesis of sepsis. Annu Rev Pathol. 2011;6:19-48.

3. Faix JD. Biomarkers of sepsis. Crit Rev Clin Lab Sci. 2013;50(1):23-36.

4. Tang BM, McLean AS, Dawes IW, Huang SJ, Lin RC. Gene-expression profiling of peripheral blood mononuclear cells in sepsis. Crit Care Med. 2009;37(3):882-8.

5. Dickinson P, Smith CL, Forster T, Craigon M, Ross AJ, Khondoker MR, et al. Whole blood gene expression profiling of neonates with confirmed bacterial sepsis. Genom Data. 2014;3:41-8.

6. Demaret J, Venet F, Friggeri A, Cazalis MA, Plassais J, Jallades L, et al. Marked alterations of neutrophil functions during sepsis-induced immunosuppression. J Leukoc Biol. 2015;98(6):1081-90.

7. Wong HR, Cvijanovich N, Allen GL, Lin R, Anas N, Meyer K, et al. Genomic expression profiling across the pediatric systemic inflammatory response syndrome, sepsis, and septic shock spectrum. Crit Care Med. 2009;37(5):1558-66.

8. R core team. A language and environment for statistical computing. http://www.r-project.org/. Accessed 26 June 2017.

9. Gentleman RC, Carey VJ, Bates DM, Bolstad B, Dettling M, Dudoit S, et al. Bioconductor: open software development for computational biology and bioinformatics. Genome Biol. 2004;5(10):R80

10. da Huang W, Sherman BT, Lempicki RA. Systematic and integrative analysis of large gene lists using DAVID bioinformatics resources. Nat Protoc. 2009:4(1):44-57.

11. da Huang W, Sherman BT, Lempicki RA. Bioinformatics enrichment tools: paths toward the comprehensive functional analysis of large gene lists. Nucleic Acids Res. 2009;37(1):1-13.

12. Shannon P, Markiel A, Ozier O, Baliga NS, Wang JT, Ramage D, et al. Cytoscape: a software environment for integrated models of biomolecular interaction networks. Genome Res. 2003;13(11):2498-504. 
13. Zhang B, Kirov S, Snoddy J. WebGestalt: an integrated system for exploring gene sets in various biological contexts. Nucleic Acids Res. 2005;33(Web Server issue):W741-8.

14. Gene Expression Omnibus. http://www.ncbi.nlm.nih.gov/geo/. Accessed 26 June 2017.

15. Gautier L, Cope L, Bolstad BM, Irizarry RA. Affy-analysis of Affymetrix GeneChip data at the probe level. Bioinformatics. 2004;20(3):307-15.

16. Langfelder P, Horvath S. WGCNA: an R package for weighted correlation network analysis. BMC Bioinform. 2008;9:559.

17. Falcon S, Gentleman R. Using GOstats to test gene lists for $\mathrm{GO}$ term association. Bioinformatics. 2007:23(2):257-8

18. Kanehisa M, Goto S. KEGG: kyoto encyclopedia of genes and genomes. Nucleic Acids Res. 2000;28(1):27-30.

19. Robin X, Turck N, Hainard A, Tiberti N, Lisacek F, Sanchez JC, et al. pROC: an open-source package for $\mathrm{R}$ and $\mathrm{S}+$ to analyze and compare ROC curves. BMC Bioinform. 2011;12:77.

20. Joly S, Rhea L, Volk P, Moreland JG, Dunnwald M. Interferon regulatory factor 6 has a protective role in the host response to endotoxic shock. PLoS ONE. 2016;11(4):e0152385.

21. Zhang L, Cardinal JS, Pan P, Rosborough BR, Chang Y, Yan W, et al. Splenocyte apoptosis and autophagy is mediated by interferon regulatory factor 1 during murine endotoxemia. Shock. 2012;37(5):511-7.

22. Gonda TJ, Ramsay RG. Adenoid cystic carcinoma can be driven by MYB or MYBL1 rearrangements: new insights into MYB and tumor biology. Cancer Discov. 2016;6(2):125-7.

23. Ramkissoon LA, Horowitz PM, Craig JM, Ramkissoon SH, Rich BE, Schumacher SE, et al. Genomic analysis of diffuse pediatric low-grade gliomas identifies recurrent oncogenic truncating rearrangements in the transcription factor MYBL1. Proc Natl Acad Sci USA. 2013;110(20):8188-93.

24. Ibegbu CC, Xu YX, Harris W, Maggio D, Miller JD, Kourtis AP. Expression of killer cell lectin-like receptor G1 on antigen-specific human CD8+ T lymphocytes during active, latent, and resolved infection and its relation with CD57. J Immunol. 2005:174(10):6088-94.

25. Henson SM, Akbar AN. KLRG1-more than a marker for T cell senescence. Age. 2009;31(4):285-91.
26. Voehringer D, Koschella M, Pircher H. Lack of proliferative capacity of human effector and memory T cells expressing killer cell lectinlike receptor G1 (KLRG1). Blood. 2002;100(10):3698-702.

27. Li L, Wan S, Tao K, Wang G, Zhao E. KLRG1 restricts memory T cell antitumor immunity. Oncotarget. 2016;7(38):61670-8.

28. Snyers L, Umlauf E, Prohaska R. Association of stomatin with lipid-protein complexes in the plasma membrane and the endocytic compartment. Eur J Cell Biol. 1999;78(11):802-12.

29. Salzer U, Prohaska R. Stomatin, flotillin-1, and flotillin-2 are major integral proteins of erythrocyte lipid rafts. Blood. 2001;97(4):1141-3.

30. Mairhofer M, Steiner M, Mosgoeller W, Prohaska R, Salzer U. Stomatin is a major lipid-raft component of platelet alpha granules. Blood. 2002;100(3):897-904

31. Arkhipova KA, Sheyderman AN, Laktionov KK, Mochalnikova WV, Zborovskaya IB. Simultaneous expression of flotillin-1, flotillin-2, stomatin and caveolin-1 in non-small cell lung cancer and soft tissue sarcomas. BMC Cancer. 2014;14:100.

32. Chen CY, Yang CY, Chen YC, Shih CW, Lo SS, Lin CH. Decreased expression of stomatin predicts poor prognosis in HER2-positive breast cancer. BMC Cancer. 2016;16:697.

33. Sanyal R, Polyak MJ, Zuccolo J, Puri M, Deng L, Roberts L, et al. MS4A4A: a novel cell surface marker for M2 macrophages and plasma cells. Immunol Cell Biol. 2017. https://doi.org/10.1038/icb.2017.18.

34. Allen M, Zou F, Chai HS, Younkin CS, Crook J, Pankratz VS, et al. Novel late-onset Alzheimer disease loci variants associate with brain gene expression. Neurology. 2012;79(3):221-8.

35. Sweeney TE, Shidham A, Wong HR, Khatri P. A comprehensive timecourse-based multicohort analysis of sepsis and sterile inflammation reveals a robust diagnostic gene set. Sci Transl Med. 2015;7(287):287ra71.

36. Maat M, Buysse CM, Emonts M, Spanjaard L, Joosten KF, de Groot R, et al. Improved survival of children with sepsis and purpura: effects of age, gender, and era. Crit Care. 2007;11(5):R112.

\section{Submit your next manuscript to BioMed Central and we will help you at every step:}

- We accept pre-submission inquiries

- Our selector tool helps you to find the most relevant journal

- We provide round the clock customer support

- Convenient online submission

- Thorough peer review

- Inclusion in PubMed and all major indexing services

- Maximum visibility for your research

Submit your manuscript at www.biomedcentral.com/submit
O Biomed Central 